\title{
Time to event analysis in the presence of competing risks
}

\author{
Inmaculada Aban, $\mathrm{PhD}^{\mathrm{a}}$ \\ a Department of Biostatistics, University of Alabama at Birmingham, Birmingham, AL
}

doi:10.1007/s12350-014-0022-2

\section{See related article, pp. 453-465}

Time to event (e.g., death or hospitalization) is a typical outcome of interest in medical research. It is not uncommon to see researchers use all-cause mortality as event of interest, consequently, treating various causes of death the same way. Standard methods are applied to analyze such outcomes: Kaplan-Meier curve to display the estimated survival curve, log rank test to compare survival curves of independent groups, and Cox proportional hazards to model the relationship between outcome and a set of predictor variables and covariates. Time to event for some subjects may not be observed due to end of study or because the subject dropped out. These observations are known to be right-censored and contain the information that the unknown event time is past the observed censored time.

Even if death is the outcome of interest, there may be cases where all-cause mortality may not be an appropriate outcome. Instead researchers are only interested in certain causes of death. The paper by Romero-Farina et al published in this issue is a good illustration. ${ }^{1}$ The authors aim to determine if there is a difference between men and women with normal myocardial perfusion gated SPECT with regard to the prognoses of these patients using stress test results. The events of interest are cardiac death, non-fatal myocardial infarction (MI), and cardiac revascularization (CR). Non-cardiac death is considered as a competing risk and not a censoring event because it precludes the occurrence of cardiac death, MI and CR while censoring event only precludes observing the time of the event. Figure 1

Reprint requests: Inmaculada Aban, PhD, Department of Biostatistics, University of Alabama at Birmingham, 1720 Second Avenue South, Birmingham, AL 35294-0022; caban@uab.edu

J Nucl Cardiol 2015;22:466-7.

$1071-3581 / \$ 34.00$

Copyright (c) 2014 American Society of Nuclear Cardiology. depicts the competing risk for this particular example. Figure 1 can easily be expanded to add more competing events.

Survival function and hazard function are important quantities in the analysis of time-to-event data. Survival function quantifies the probability of a person being event free at a given point in time while hazard function quantifies the risk that a person who is event-free at a given point in time will experience the event in the next instant. Kaplan-Meier curve is commonly used for estimating the survival function, and Cox proportional hazards model is commonly used to model the association of the hazard function with a set of predictor variables or covariates.

In the presence of competing risks, each competing event has an associated hazard function known as the cause-specific hazards. Akin to the regular hazard function, a cause-specific hazard quantifies the risk of experiencing an event from a particular cause. Associated with this cause-specific hazard is the cumulative incidence function (CIF), also known as subdistribution function, which quantifies the probability of experiencing an event on or before a given point in time due to a particular cause.

How then do we analyze the time to event data when competing events are present? One approach is to treat the competing event as a censoring event and use standard time-to-event methods for analyses. However, standard methods assume that the time to the primary event and the time to censoring are independent. In other words, the hazard at a given time point of subjects who have not experienced the primary event is the same as the hazard of those who have experienced the competing event. This is clearly violated if the competing event prevents the subject from experiencing the primary event. In the case where the competing event is not death (for example, stroke) and the primary event may still occur, the risk of the primary event may be altered after experiencing the competing event (e.g., experiencing a stroke may increase the risk of experiencing MI). As a consequence, the Kaplan-Meier curve which 


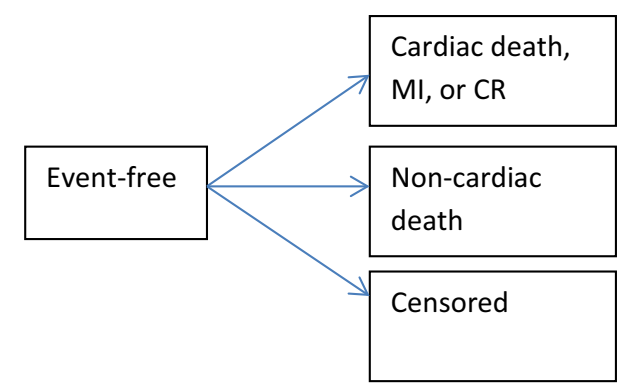

Figure 1. Competing risk diagram.

is the standard and the most basic estimation tool for the survival probabilities, will be biased and uninterpretable. ${ }^{2}$

A more appropriate approach is to work with CIF as this quantity by definition is cause-specific. Moreover, CIF does not assume independence of competing events. Therefore, estimating CIF based on the data for each competing event is appropriate and easily interpreted. Instead of plotting the Kaplan-Meier curve for the primary event, one will plot the estimated CIF. In comparing CIFs of different groups, Gray $^{2}$ proposed a log-rank type of test which is an analog of the standard log-rank tests to compare Kaplan-Meier curves.

To address competing risks in regression modeling, one approach may be to use Cox proportional hazards model to model the cause-specific hazards. However, testing for equality of cause-specific hazards is not equivalent to testing the equality of CIF. ${ }^{3}$ Consequently, it is possible, in the extreme case, that a particular predictor variable or covariate may be strongly associated with the cause-specific hazard but is not associated with the CIF. ${ }^{4}$ Fine and Gray proposed a proportional hazards model for subdistribution that would more appropriately model the effect of predictors or covariates on the CIF.

In the paper by Romero-Farina et al, the authors used both Cox proportional hazards model and Fine and
Time to event analysis in the presence of competing risks

Gray model to investigate the differences in the time to primary outcome (cardiac death, MI and CR) in the presence of non-cardiac deaths and censoring. Although in their analyses, both models arrived at the same conclusions, this will not always be true. Therefore, caution should be taken in choosing the proper analyses. Since the primary event of interest is a composite outcome, it would have been interesting if the authors break this composite outcome and estimated the CIF separately for cardiac death, MI and CR to determine if the group differences are being driven by a certain outcome.

In summary, competing events are commonly encountered in medical research when time-to-event is the outcome. Using standard methods of analyses in the presence of competing events may no longer be appropriate. Instead methods based on CIF or subdistribution functions must be considered. [For more details, readers may refer to a tutorial paper by Putter, Fiocco and Geskus ${ }^{5}$ which includes discussions on the implementation of these methods using existing statistical softwares.]

\section{References}

1. Romero-Farina G, Candell-Riera J, Ferreira-González I, AguadéBruix S, Pizzi N, García-Dorado D. Normal myocardial perfusion gated SPECT and positive stress test: Different prognoses in women and men. J Nucl Cardiol 2014. doi:10.1007/s12350-014-0009-z.

2. Gooley TA, Leisenring W, Crowley J, Storer BE. Estimation of failure probabilities in the presence of competing risks: New representations of old estimators. Stat Med 1999;18:695-706.

3. Gray RJ. A class of k-sample tests for comparing the cumulative incidence of a competing risk. Ann Stat 1988;16:1141-54.

4. Fine JP, Gray RJ. A proportional hazards model for the subdistribution of a competing risk. J Am Stat Assoc 1999;94:496-509.

5. Putter H, Fiocco M, Geskus RB. Tutorial in biostatistics: Competing risks and multi-state models. Stat Med 2007;26:2389-430. doi: $10.1002 / \operatorname{sim} .2712$. 